\title{
Contraceção e risco de tromboembolismo venoso: um estudo caso-controlo
}

Ana Catarina Guimarães (MD), ${ }^{1-2}$ Patrício Costa (Ph.D), ${ }^{1-2}$ Afonso Rocha (MD), ${ }^{3}$ Fátima Queirós (MD), ${ }^{4}$ Ana Paula Barbosa (MD), ${ }^{5}$ Cristina Nogueira-Silva (MD, Ph.D) $)^{1-3}$

\section{RESUMO}

Objetivos: Comparar o risco de tromboembolismo venoso (TEV) entre a $4^{a}$ geração e as gerações anteriores de contracetivos orais combinados (COC); analisar a correlação existente entre a idade, índice de massa corporal (IMC) e duração da toma do COC e o risco de TEV.

Tipo de estudo: Estudo caso-controlo.

Local: Centro Hospitalar do Alto-Ave, Hospital de Braga e Unidade Local de Saúde do Alto Minho.

População: Foram estudadas no total 257 mulheres, das quais 122 tinham diagnóstico prévio de TEV e 135 não tinham história de TEV. Métodos: Reviram-se os registos clínicos de mulheres em seguimento por TEV, ocorrido entre 2010 e 2013. As mulheres a tomar COC aquando do evento tromboembólico eram os casos. Os controlos foram as mulheres seguidas em consulta de planeamento familiar, a tomar COC e sem antecedentes de TEV.

Resultados: Os COC de $4^{\text {a }}$ geração não aumentam o risco de TEV comparativamente com as gerações anteriores (OR = 1,77; IC95 $0,93-3,38 ; p=0,083)$. Não se observou correlação entre o IMC e o risco de TEV (OR = 1,53; IC95 0,76-3,06; $p=0,234)$. Por outro lado, a idade (OR = 1,5; IC95 1,02-1,09; $p=0,001)$ e a duração da toma do COC (OR = 2,41; IC95 1,19-4,87; $p=0,014)$ estiveram associados a risco de TEV.

Conclusões: O risco de TEV depende da idade e duração do uso do COC. Tendo em consideração as limitações do estudo, a interpretação dos resultados deve ser cautelosa, quanto à segurança dos $\operatorname{COC}$ de $4^{\mathrm{a}}$ geração.

Palavras-chave: Contracetivos Orais Combinados; Progestativo; Tromboembolismo Venoso.

\section{INTRODUÇÃO}

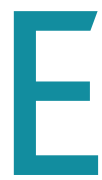

$\mathrm{m}$ Portugal, de acordo com o $4^{\circ}$ Inquérito Nacional de Saúde (2009), 85,4\% da população feminina em idade reprodutiva utiliza um método de contraceção. Destes, 65,9\% dos casos correspondem aos contracetivos orais. ${ }^{1}$ Existem dois tipos de contracetivos orais: os contracetivos orais combinados (COC), que contêm estroprogestativos, e as pílulas constituídas apenas por progestativo. ${ }^{1-2}$ Atualmente, os COC são utilizados por um número estimado de 100 milhões de mulheres em todo o mundo. ${ }^{3}$

O risco de fenómenos tromboembólicos (TEV), venosos e arteriais, com o uso de COC, foi reportado pela primeira vez em 1961 e foi associado à elevada dosagem de estrogénios (>50 $\mu \mathrm{g}$ ), levando à sua redução para $30 \mu \mathrm{g} .{ }^{4}$ Assim, a dose de estrogénios nestas formulações é um fator crucial para a obtenção dos benefícios para os quais são administrados e, de igual forma, para os riscos e efeitos adversos. A incidência de TEV aumenta com a idade, sendo que nas mulheres com me- nos de 40 anos o risco anual é de 1 em 10.000 (risco este muito provavelmente subestimado). A incidência nas utilizadoras de COC é, contudo, cinco a seis vezes superior. ${ }^{5}$

Atualmente, em Portugal, as dosagens de estrogénios contidos nos contracetivos orais comercializadas variam entre 15 e $35 \mu \mathrm{g} .{ }^{1}$ Os estrogénios utilizados nestas formulações são o etinilestradiol e o valerato de estradiol. ${ }^{1}$ Por outro lado, os

1. Instituto de Ciências da Vida e da Saúde (ICVS), Escola de Ciências da Saúde, Universidade do Minho, Braga, Portugal.

2. ICVS/3B's - Laboratório Associado do Governo Português, Braga/Guimarães, Portugal.

3. Serviço de Ginecologia e Obstetrícia do Hospital de Braga, Braga, Portugal. 4. Serviço de Imuno-hemoterapia do Centro Hospitalar do Alto Ave, E.P.E. - Guimarães/Fafe, Portugal.

5. Serviço de Imuno-hemoterapia do Hospital de Braga, Braga, Portugal. Ana Catarina Guimarães: Aluna do $6^{\circ}$ ano do Mestrado Integrado em Medicina Patrício Costa: Professor Auxiliar

Afonso Rocha: Assistente Graduado de Ginecologia e Obstetrícia Fátima Queirós: Assistente Graduada de Imuno-Hemoterapia

Ana Paula Barbosa: Especialista em Imuno-hemoterapia com o grau de Consultor Cristina Nogueira-Silva: Médica Interna de Ginecologia e Obstetrícia; Professora Auxiliar Convidada a 50\% 
COC são também caracterizados em gerações, tendo em conta o tipo de progestativo utilizado. Os COC de $2^{\mathrm{a}}$ geração são constituídos por levonorgestrel ou, menos frequentemente, por norgestimato; a $3^{\text {a }}$ geração é constituída por gestodeno ou desogestrel; e, mais recentemente, foram introduzidos novos progestativos referidos como de $4^{\text {a }}$ geração: a drosperinona e o dienogest. ${ }^{1,4,6}$ As últimas gerações, embora mais caras, ganharam popularidade, sobretudo entre as mulheres mais jovens, pela associação da atividade antimineralocorticóide à ação antiandrogénica. ${ }^{1,6}$ Porém, pouco depois da sua introdução, foram e têm sido publicados estudos quanto ao risco de TEV associado aos contracetivos de $4^{\text {a }}$ geração comparativamente com os das gerações anteriores.

Recentemente, foram publicados dois trabalhos que reportaram um maior risco de TEV associado às pílulas que contêm drosperinona na sua constituição. Este risco é, segundo os estudos, duas vezes superior comparativamente com as pílulas constituídas por levonorgestrel (2a geração).4,7 Porém, outros estudos não corroboraram esse aumento do risco. ${ }^{8-9}$ De facto, as publicações sobre este tema têm sido controversas e algo divergentes..$^{8-9}$ Desta forma, autoridades reguladoras como a Food and Drug Administration, European Medicines Agencye Therapeutic Goods Administration encontram-se atualmente a rever os estudos epidemiológicos referentes a este tema a fim de alcançar uma conclusão relativamente à segurança da administração dos contracetivos de $4^{\text {a }}$ geração. Entretanto, e por este motivo, foi lançado um conjunto de alertas..$^{10-12} \mathrm{Na}$ Austrália apenas os contracetivos de $2^{\text {a }}$ geração são comparticipados e o governo francês anunciou, em janeiro de 2013, a cessação da comparticipação das pílulas de $3^{\mathrm{a}} \mathrm{e} 4^{\mathrm{a}}$ gerações. ${ }^{11}$ Da mesma forma, em Portugal, em janeiro de 2014, foi dirigido aos profissionais de saúde um comunicado a alertar para as diferenças entre os diferentes tipos de COC relativamente ao risco de tromboembolismo. ${ }^{13}$ Este baseou-se nos resultados de uma revisão de segurança realizada em toda a Europa, referindo que dentro dos contracetivos disponíveis atualmente, os de $2^{\text {a }}$ geração são os que possuem menor risco de TEV ${ }^{13}$

Foi neste contexto de controvérsia que nos propusemos desenvolver este trabalho. Sendo a eficácia na contraceção igual, independentemente do tipo de progestativo, a escolha do contracetivo deverá basear-se na incidência dos efeitos adversos, visto que, como são utilizados por um grande número de mulheres, o mínimo aumento destes efeitos acaba por afetar um número significativo desta população. Assim, foi nosso objetivo principal estimar o risco de TEV associado à $4^{\mathrm{a}}$ geração em comparação às que fazem COC de gerações anteriores.

\section{MÉTODOS}

Trata-se de um estudo observacional, de caso-controlo, realizado no Centro Hospitalar do Alto-Ave, Hospital de Braga e Unidade Local de Saúde do Alto Minho.

A dimensão amostral foi obtida considerando uma perspetiva mais conservadora, com o objetivo de obter mais de 50 participantes por cada preditor incluído no modelo.

A amostra em estudo foi selecionada por um método de amostragem por conveniência. A amostra de casos foi conseguida através da análise de processos clínicos de mulheres em seguimento em consulta de Imuno-hemoterapia por episódio de TEV ocorrido no período entre janeiro de 2010 e setembro de 2013. De seguida, selecionaram-se as mulheres em idade reprodutiva e a tomar COC na altura do evento tromboembólico. Foram excluídos do estudo todos os casos em que faltasse informação relativa aos parâmetros a avaliar, mais frequentemente o nome comercial/princípio ativo do contracetivo. Foram igualmente recolhidos dados relativos a fatores de risco para ocorrência de TEV, como: idade, índice de massa corporal (IMC), geração de COC e duração da sua toma aquando da ocorrência do TEV, bem como antecedentes pessoais e familiares de relevo para o estudo, como episódios prévios de TEV.

A amostra dos controlos foi selecionada através de um processo de amostragem de conveniência, com uma lógica de 1:1, através da consulta dos processos clínicos informatizados de pacientes em consulta de Planeamento Familiar de Ginecologia/Obstetrícia. A escolha da consulta destes processos foi decidida pelo facto de nestes existir maior probabilidade de referência ao tipo de contracetivo usado por mulheres em idade reprodutiva, aparentemente saudáveis, sem história de TEV.

Foram critérios de inclusão dos casos: i) mulheres em idade reprodutiva; e ii) mulheres a tomar $\operatorname{COC}\left(2^{\mathrm{a}}, 3^{\mathrm{a}}\right.$ ou $4^{\mathrm{a}}$ gerações) e com diagnóstico de TEV. Por sua vez, foram critérios de inclusão dos controlos: mulheres em idade reprodutiva e a tomar COC (2a $3^{\mathrm{a}}$ e $4^{\mathrm{a}}$ gerações). Foram critérios de exclusão do estudo i) mulheres com diagnóstico de tromboembolismo arterial; ii) mulheres que não tomavam COC (2a, $3^{\mathrm{a}}$ ou $4^{\mathrm{a}}$ gerações); iii) mulheres com diagnóstico de trombofilia; iv) grávidas ou puérperas; v) mulheres com doenças crónicas (por exemplo, neoplasias).

O estudo foi aprovado pelas Comissões Executivas e de 
Ética dos referidos hospitais e a colheita de dados foi realizada nos meses de julho, agosto e setembro do ano de 2013.

Os dados foram introduzidos numa base de dados construída em SPSS ${ }^{\circledR}$ (Statistical Package for the Social Sciences, versão 22, Chicago, USA). Na análise estatística foi utilizado o mesmo programa. Inicialmente procedeu-se a uma análise descritiva das amostras em estudo, casos e controlos, tendo-se posteriormente caracterizado a amostra em função das gerações de COC em questão. Verificada a normalidade com os testes Kolmogorov-Smirnove Shapiro-Wilk, recorreu-se ao teste $t$-student para amostras independentes para comparar médias de variáveis quantitativas. Para as restantes variáveis utilizaram-se testes não paramétricos, como o teste qui-quadrado para comparação entre as variáveis qualitativas. Da mesma forma, através da regressão logística binária, procedemos à comparação do Odds Ratio (OR) de TEV entre a $4^{\text {a }}$ geração e as gerações anteriores de COC, assim como à análise da existência de uma associação estatisticamente significativa entre as variáveis idade, IMC e duração da toma do COC e o risco de TEV.

Pelo facto de a informação relativa aos antecedentes pessoais e familiares só ter sido conseguida no grupo dos casos, esta não pôde ser utilizada no tratamento dos dados. Admitiu-se significância estatística para valores de $p<0,05$.

\section{RESULTADOS}

Foram colhidos dados de 257 mulheres (122 casos e 135 controlos), em idade reprodutiva e a tomar COC, nos três hospitais que colaboraram no estudo, durante o período previsto no mesmo. No grupo dos casos, 29 (23,8\%) mulheres tomavam contracetivos de $4^{\text {a }}$ geração e 93 de gerações anteriores, enquanto no grupo controlo $22(16,3 \%)$ mulheres tomavam contracetivos de $4^{a}$ geração e 113 de gerações anteriores. Das 257 mulheres, 122 são pacientes que sofreram pelo menos um episódio de TEV: 75 (61,5\%) sofreram trombose venosa profunda, $32(26,2 \%)$ tromboembolismo pulmonar, $9(7,4 \%)$ trombose dos seios venosos e $6(4,9 \%)$ trombose da veia porta. Os controlos são constituídos por 135 mulheres, sem antecedentes de TEV.

No que diz respeito às características da amostra em estudo, a idade média dos casos foi de 36,19 \pm 9,80, variando dos 15 aos 53 anos. Por sua vez, a idade média dos controlos foi de 33,21 $\pm 7,00$, variando dos 16 anos aos 47 anos.

Procedeu-se a uma análise das características da amostra em estudo, em função da geração de COC que tomavam. A idade média no grupo de mulheres a tomar COC da $4^{\text {a }}$ geração foi de 33,00 \pm 8,63, enquanto nas gerações anteriores foi de 35,02 $\pm 8,51$. A percentagem de mulheres obesas $\left(\text { IMC } \geq 30 \mathrm{Kg} / \mathrm{m}^{2}\right)^{14}$ no grupo que tomava a $4^{\text {a }}$ geração foi de $11,8 \%$ e nas gerações anteriores de $17 \%$. A percentagem de mulheres do grupo da 4 a geração com duração da toma de contracetivo igual ou inferior a um ano foi de $23,5 \%$, enquanto no grupo das gerações anteriores foi de $16 \%$.

No Quadro I encontra-se apresentada a associação das variáveis independentes com a ocorrência de TEV (variável dependente). De notar que, com a exceção da variável idade, todas as outras variáveis representadas são dicotómicas. Como se pode observar, quanto maior for a idade maior a probabilidade de ocorrência de TEV (OR 1,05; $\mathrm{IC}_{95}$ 1,02-1,09 - Quadro I). As mulheres com uma duração da toma do COC por um tempo igual ou inferior a um ano apresentam 2,4 vezes maior risco de ocorrência de TEV do que as mulheres com uma duração da toma superior a um ano (OR 2,41; $\mathrm{IC}_{95}$ 1,19-4,87; $p=0,014$ - Quadro I). Assim, determinaram-se como preditores do risco de TEV a idade e a duração da toma do COC por um período igual ou inferior a um ano. Contrariamente, as mulheres a tomar COC da $4^{\mathrm{a}}$ geração não apresentaram associação com um risco superior de TEV comparativamente com as mulheres a tomar as gerações anteriores (OR 1,77; $\mathrm{IC}_{95} 0,93-3,38 ; p=0,083$ - Quadro I). Igualmente, as mulheres obesas não apresentaram associação com um maior risco de TEV (OR 1,53; $\mathrm{IC}_{95}$ 0,763,06; $p=0,234$-Quadro I). Determinamos, assim, que neste estudo nem a geração de COC nem o IMC são preditores da ocorrência TEV. Os resultados de OR bruto para estas variáveis não se revelou significativo (Quadro I).

Foram verificados e cumpridos os principais requisitos para a aplicação da regressão logística binária.

\section{DISCUSSÃO}

A idade média no grupo de mulheres a tomar COC da $4^{\mathrm{a}}$ geração $(33,00 \pm 8,63)$ foi inferior à das a fazer gerações anteriores $(35,02 \pm 8,51)$. A percentagem de mulheres obesas $\left(\mathrm{IMC} \geq 30 \mathrm{Kg} / \mathrm{m}^{2}\right)^{14}$ foi inferior no grupo que tomava a $4^{\text {a }}$ geração ( $11,8 \%$ s. $17 \%)$ e a duração da toma de contracetivo igual ou inferior a um ano foi superior no grupo da $4^{\text {a }}$ geração (23,5\% vs. $16 \%)$. Como se pôde observar, a ocorrência de TEV esteve associado à idade (OR 1,05; $\mathrm{IC}_{95}$ 1,02-1,09; $p$ $=0,001$-Quadro I) e à duração da toma do COC por um tempo igual ou inferior a um ano (OR 2,41; $\mathrm{IC}_{95} 1,19-4,87 ; p=$ 0,014 - Quadro I).

Neste estudo não se verificou associação entre risco de 
TEV e a toma de COC da $4^{\mathrm{a}}$ geração (OR 1,77; $\mathrm{IC}_{95}$ 0,93-3,38; $p=$ 0,083 - Quadro I) ou a obesidade (OR 1,53; IC $_{95}$ 0,76-3,06; $p=0,234-$ Quadro I). Este trabalho não demonstrou risco acrescido de TEV associado à utilização de COC de $4^{\text {a }}$ geração comparativamente com os de gerações anteriores $\left(2^{\mathrm{a}}\right.$ e $3^{\text {a }}$ gerações). Este resultado vai, assim, ao encontro de estudos prévios que reportaram que os COC de $4^{\text {a }}$ geração não se assoQUADRO I. Associação entre as variáveis selecionadas (variáveis independentes) e a ocorrência de TEV (variável dependente), através da utilização da regressão logística binária

\begin{tabular}{|l|c|c|c|c} 
Fatores de Risco & OR bruto & IC 95\% & OR ajustado & IC 95\% \\
\hline $\mathbf{4}^{\text {a }}$ geração de COC & 1,60 & $0,86-2,97$ & 1,77 & $0,93-3,38$ \\
Idade & $1,04^{*}$ & $1,01-1,07$ & $1,05 \dagger$ & $1,02-1,09$ \\
IMC $\geq \mathbf{3 0} \mathbf{~ K g} / \mathbf{m}^{2}$ & 1,51 & $0,77-2,96$ & 1,53 & $0,76-3,06$ \\
Duração da toma do COC $\leq \mathbf{1}$ ano & $2,07^{*}$ & $1,07-4,00$ & $2,41^{*}$ & $1,19-4,87$
\end{tabular}

COC: Contracetivo oral combinado; IMC: Índice de massa corporal; TEV: Tromboembolismo venoso. ${ }^{*} p<0,05 ; \uparrow p<0,001$.

ciam a aumento do risco de TEV em comparação com as outras gerações estudadas..$^{9,14-16}$

De facto, outros estudos têm demonstrado que o risco de TEV varia consoante o tipo de progestativo utilizado. ${ }^{17-18}$ Disto são exemplo dois estudos publicados recentemente que reportaram a associação dos COC de 4a geração com o dobro do risco de TEV.-5

Foi possível confirmar com o presente estudo que a idade é, efetivamente, um preditor do risco para a ocorrência de eventos tromboembólicos venosos ( $p=0,001)$, ou seja, a incidência de TEV aumenta com a idade. ${ }^{4,6,14,19}$

A não comprovação do efeito da obesidade no risco de TEV ( $p=0,234$ ), eventualmente por falta de poder amostral, contraria a maioria dos estudos realizados que demonstraram que a obesidade é um fator que predispõe ao TEV. ${ }^{6,20}$

Quanto à duração da toma do COC, os resultados vieram de encontro aos dados da literatura, em que o risco de TEV é maior durante o primeiro ano de uso do mesmo $(p=0,014)$, diminuindo o risco com a duração do uso, à semelhança do descrito na literatura. ${ }^{4-5}$

Os eventos tromboembólicos arteriais foram excluídos do presente estudo, visto tratar-se de um risco muito baixo na faixa etária estudada e por se desconhecer que este aumente com algum progestativo em particular. ${ }^{21}$

Sendo a metodologia adotada a principal limitação deste trabalho, foi a possível tendo em consideração o tempo e recursos humanos disponíveis. Salientam-se como limitações relevantes:

- Os dados obtidos dependerem maioritariamente dos registos informatizados dos processos clínicos, tornando-nos dependentes de um registo completo.

- A amostragem ter sido de conveniência.

- A ausência de uma clara quantificação da toma dos con- tracetivos que permitisse determinar o tempo até ao evento.

- A ausência de quantificação das mulheres excluídas por eventos tromboembólicos venosos e a fazer contracetivos hormonais combinados por falta de informação.

Salientamos, porém, que estes dados foram alvo de uma análise multivariada, o que, de alguma forma, permitiu controlar estatisticamente o viés de confundimento.

De facto, este tema permanece controverso, mas de relevância extrema. Assim, urgem estudos mais robustos e eventuais revisões sistemáticas com meta-análise.

Em conclusão, tendo presente as limitações inerentes, o nosso estudo não demonstrou evidência de que possamos afirmar a superioridade do perfil de segurança entre gerações de COC. Contudo, confirmaram--se como preditores do risco de TEV a idade e a duração da toma do COC por um período igual ou inferior a um ano.

\section{REFERÊNCIAS BIBLIOGRÁFICAS}

1. Sociedade Portuguesa de Ginecologia, Sociedade Portuguesa da Contracepção, Sociedade Portuguesa de Medicina da Reprodução. Consenso sobre contracepção 2011. Lisboa: Edições First News; 2011.

2. Direção-Geral da Saúde. Saúde reprodutiva, planeamento familiar. Ed rev. Lisboa: DGS; 2008. ISBN 9789726751823

3. Hannaford P. Health consequences of combined oral contraceptives. Br Med Bull. 2000;56(3):749-60.

4. Van Hylckama Vlieg A, Helmerhorst FM, Vandenbroucke JP, Doggen CJ, Rosendaal FR. The venous thrombotic risk of oral contraceptives, effects of oestrogen dose and progestogen type: results of the MEGA case-control study. BMJ. 2009;339:b2921.

5. Rott H. Thrombotic risks of oral contraceptives. Curr Opin Obstet Gynecol. 2012;24(4):235-40.

6. Petitti DB. Clinical practice: combination estrogen-progestin oral contraceptives. N Engl J Med. 2003;349(15):1443-50.

7. Lidegaard O, Lokkegaard E, Svendsen AL, Agger C. Hormonal contraception and risk of venous thromboembolism: national follow-up study. BMJ. 2009;339:b2890. 
8. Shapiro S, Dinger J. Risk of venous thromboembolism among users of oral contraceptives: a review of two recently published studies. J Fam Plann Reprod Health Care. 2010;36(1):33-8.

9. Fruzzetti F, Perini D, Spirito N, Manca R. Pillola e rischio trombotico: gli studi epidemiologici [Oral contraceptive pill and thrombotic risk: epidemiological studies] Minerva Ginecol. 2012;64(6):539-49. Italian

10. Food and Drug Administration. FDA-funded study: combined hormonal contraceptives (CHCs) and the risk of thromboembolism and other cardiovascular events. FDA 2011 Mar 3 [cited 2014 Jan 21]. Available from: http://www.fda.gov/Drugs/DrugSafety/ucm274292.htm

11. European Medicines Agency. European Medicines Agency to review third- and fourthgeneration combined oral contraceptives. EMA; 2013 Jan 28 [cited 2014 Jan 21].Available from:http://www.ema.europa.eu/ema/index.jsp?curl=pages/news_and_events/ news/2013/01/news_detail_001700.jsp\&mid=WC0b01ac058004d5c1

12. Therapeutic Goods Administration. Oral contraceptives containing drospirenone (Yaz and Yasmin). Department of Health,Australian Government; 2011 [cited 2014 Jan 20]. Available from: http://www.tga.gov.au/safety/alerts-medicine-oral-contraceptives-110706.htm

13. INFARMED. Comunicação dirigida aos profissionais de saúde. Lisboa: INFARMED; 2014 Jan 22 [cited 2014 Feb 14]. Available from: http://www.infarmed.pt/portal/page/portal/INFARMED/MEDICAMENTOS_USO_HUMANO/FARMACOVIGILANCIA/INFORMACAO_SEGURANCA/CARTAS_PROFISSIONAIS/Tab/DHPC_ CHC_final_22012014.pdf

14. World Health Organization. Obesity: preventing and managing the global epidemic. Geneva:WHO; 2000 [cited 2014 Jan 20]. Available from: http://www.who.int/nutrition/publications/obesity_executive_summary.pdf

15. Jick SS, Hernandez RK. Risk of non-fatal venous thromboembolism in women using oral contraceptives containing drospirenone compared with women using oral contraceptives containing levonorgestrel: case-control study using United States claims data. BMJ. 2011;342:d2151
16. Brown DA, Vartan CM. Risk of venous thromboembolism with drospirenone-containing oral contraceptives. Am J Health Syst Pharm. 2011;68(11):1003-10.

17. Dinger J, Assmann A, Möhner S, Mihn TD. Risk of venous thromboembolism and the use of dienogest and drospirenone containing oral contraceptives: results from a German case-control study. J Fam Plann Reprod Health Care. 2010;36(3):123-9.

18. Rott $\mathrm{H}$. Contraception, venous thrombosis and biological plausability. Minerva Med. 2013;104(2):161-7.

19. Previtali E, Bucciarelli P, Passamonti SM, Martinelli I. Risk factors for venous and arterial thrombosis. Blood Transfus. 2011;9(2):120-38.

20. Gurney EP, Murthy AS. Obesity and contraception: metabolic changes, risk of thromboembolism, use of emergency contraceptives, and role of bariatric surgery. Minerva Ginecol. 2013;65(3):279-88.

21. Wu CQ, Grandi SM, Filion KB, Abenhaim HA, Joseph L, Eisenberg MJ. Drospirenone-containing oral contraceptive pills and the risk of venous and arterial thrombosis: a systematic review. BJOG. 2013;120(7):801-10.

\section{CONFLITOS DE INTERESSE}

Os autores declaram não ter conflito de interesses.

\author{
ENDEREÇO PARA CORRESPONDÊNCIA \\ Cristina Nogueira-Silva \\ Instituto de Ciências da Vida e da Saúde (ICVS) \\ Escola de Ciências da Saúde, Universidade do Minho \\ Campus de Gualtar. 4710-057 Braga. Portugal \\ Telef.: +351 253604 909; Fax: +351 253604847 \\ Email: cristinasilva@ecsaude.uminho.pt
}

Recebido em 20-02-2014

Aceite para publicação em 18-10-2014

\section{ABSTRACT}

\section{CONTRACEPTION AND RISK OF VENOUS THROMBOEMBOLISM: A CASE-CONTROL STUDY}

Objectives: To compare the risk of venous thromboembolism (VTE) among users of fourth generation oral contraceptives and previous generations of combined oral contraceptives ( $\mathrm{COC}$ ), and to analyze the possible correlations between age, body mass index (BMI) and duration of COC use and the risk of VTE.

Study design: Case-control study.

Setting: Centro Hospitalar do Alto-Ave, Hospital de Braga and Unidade Local de Saúde do Alto Minho.

Participants: A sample of 257 women was analyzed, including 122 with a previous history of VTE and 135 without a history of VTE. Methods: The medical records of women with a history of VTE, which occurred between 2010 and 2013, were reviewed. Women of using $\mathrm{COC}$ at the time of the VTE were identified as cases. The controls were women using COC followed in a family planning clinic, without a history of VTE.

Results: Fourth generation COC do not increase the risk of VTE compared to older COC (OR = 1.77; IC95 0.93-3.38; $P=0.083)$. There was no correlation between $\mathrm{BMI}$ and the risk of VTE $(\mathrm{OR}=1.53$; IC95 0.76-3.06; $p=0.234)$. Age $(\mathrm{OR}=1.5 ; \mathrm{IC} 951.02-1.09 ; p=$ $0.001)$ and duration of COC use $(O R=2.41 ; \mathrm{IC} 951.19-4.87 ; p=0.014)$ were associated with VTE.

Conclusions: The risk of VTE depends on age and duration of COC use. Due to limitations of the study, the finding of relative safety of fourth generation $\mathrm{COC}$ for the risk of VTE compared to older contraceptives must be interpreted with caution

Keywords: Combined Oral Contraceptives; Progestins; Venous Thromboembolism. 